\title{
Innovating surgical practice and policy: lessons learnt from the second Oxford Surgical Innovation Conference
}

\author{
S. Hoppe*, M. de Andres Crespo*, M. A. Williams, A. Handa and J. McVeigh \\ *Authors contributed equally
}

${ }^{1}$ Nuffield Department of Surgical Sciences, University of Oxford, UK

\section{Introduction}

We recently read the updated Future of Surgery report by the Royal College of Surgeons. ${ }^{1}$ The section on changes to training particularly resonated with us. These key changes centred around the use of augmented reality (AR) and virtual reality (VR) in education, the idea of surgeons also being entrepreneurs and the need for surgeons to be scientists at their core in order to advance the field.

Following the success of the inaugural event last year, the second Oxford Surgical Innovation (OxSI) Conference was held at St Catherine's College on the 13th March 2020

objectives:

The OxSI Conference has two key learning

1. To educate delegates on the processes of engaging in safe and successful innovation in surgery

2. To present delegates with examples of ongoing innovation in surgery to contextualise these skills and processes.

\section{OxSI 2020}

With the proposed changes to training in Future of Surgery, we sought the following plenary speakers and workshops to inform our delegates.

Mr Tom Cosker, Director of human anatomy at the University of Oxford, showed us that AR in the form of HoloLens technologies will enable students and teachers to visualise anatomy together. This interactive method of teaching would augment anatomy learning, a crucial skill for all future surgeons, and an essential component of building a strong baseline knowledge. Likewise, VR could enable trainees to hone their operating skills before doing so on patients, an activity that would inevitably result in increased patient safety. Dr Helen Higham, consultant anaesthetist and one of the keynote speakers, focused on the advances in simulation and technology for learning in surgery, emphasising the need for VR and simulation in surgical training. At Oxford Medical School, simulation sessions are already used to teach management of emergency medical situations, and VR is currently being trialled for surgical training. Stephen Taylor, Head of the Computational Biology Research Group, led one of the breakout sessions at OxSI, introducing delegates to how VR might be used in both medical and surgical training in the future.

Our laparoscopic surgical skills competition provided an excellent way to incorporate surgical practice into the day. This was led by Mr Ioannis Gerogiannis, who gave delegates an introduction into the use of laparoscopic instruments, and then guided them through a series of tasks testing manual dexterity. This a good example of the use of simulating surgical techniques before entering theatre, and received great feedback from delegates.

Entrepreneurship is another facet of a surgical career that is rarely discussed. Professor Peter Friend, a consultant transplant surgeon, saw a potential for an innovative technology for storing transplant organs for longer. He collaborated with Professor Constantin Coussios and created OrganOx, a company that produces liver perfusion equipment to sustain liver for transplantation for 24 hours with normothermic perfusion. Professor Coussios' talk Translating Innovative Surgical Devices from Concept into Clinical Practice, gave a fascinating insight into the steps from an innovative idea, to changing surgical practice. This new technology has led to 50\% fewer discarded organs compared to previous practice, and $20 \%$ more transplanted organs.2 This innovation will change the landscape of organ transplantation, with OrganOx now developing new technologies for the transplantation of other organs.

In order to help delegates develop the skills needed to become entrepreneurs themselves, we organised two further plenary talks and two workshop sessions on the topic. This included a talk by Professor of surgical science and practice, Peter McCulloch, on the IDEAL (Idea, Development, Exploration, Assessment, Long-term followup) collaboration. This is a stepwise framework to improve research, which could then culminate in the discovery and creation of new technologies for the development of a device. The second plenary session by Dr Neil McGuire, senior clinician at the Medicines and Healthcare products Regulatory Agency (MHRA), discussed getting regulatory approval for a device. Once the device has this approval, an entrepreneur must then convert it into a company and legally patent it. These two aspects of the creation of a device were discussed in our workshops given by Mr Regent Lee and Dr Dinali de Silva, respectively.

Lastly, it is important to remember that even as 
we become more specialised as surgeons, we are all still scientists. Our final speaker, Dr Sushma Shankar, not only works as a transplant surgeon, but is also a laboratory immunologist working on creating a therapeutic treatment for cancer that would obviate the use of surgery. Building on the recent success of CAR (chimeric antigen receptor) $\mathrm{T}$ cell therapy in the treatment of leukaemia,3 Dr Shankar was looking at similarly modifying B cells to specifically target cancerous cells in the body. This is a perfect example of a divergence from a traditional surgical training pathway encouraged by the report.

\section{Conclusion}

In conclusion, the changes in training section from the Future in Surgery Report outlined key ideas for improving surgical training in the future, including the use of AR, VR and simulation, as well as increased support for surgeons to diverge from traditional surgical training pathways to undertake research and act as entrepreneurs. The Second Oxford Surgical Innovation Conference (OxSI) had similar aims to prepare delegates for these changes in surgery. Talks and workshops from world-renowned surgeons covered a vast range of topics but ultimately all gave a fascinating insight into what is needed to improve surgical practice and policy. We must develop skills in other areas to incorporate new technologies, become entrepreneurs and maintain our scientific interest if we are to develop alongside surgical careers.

\section{Acknowledgements}

Dr Helen Higham, Dr Neil McGuire, Professor Constantin Coussios, Professor Peter McCulloch, Dr Sushma Shankar, Mr Regent Lee, Mr Tom Cosker, Dr Dinali de Silva, Mr Stephen Taylor and Mr Ioannis Gerogiannis.

\section{Conflicts of interest}

None.

\section{Funding}

None.

\section{References}

1. Welcome to the Future of Surgery [Internet]. Future Of Surgery. [cited 2020 Mar 21]. Available from: https://futureofsurgery.rcseng.ac.uk

2. CMS C. OrganOx [Internet]. OrganOx. [cited 2020 Mar 21]. Available from: https://www.organox.com/index/ index

3. Pehlivan KC, Duncan BB, Lee DW. CAR-T Cell Therapy for Acute Lymphoblastic Leukemia: Transforming the Treatment of Relapsed and Refractory Disease. Curr Hematol Malig Rep. 2018;13(5):396-406. 